\title{
SYMBIOSIS
}

\section{Breaking the ice with your host}

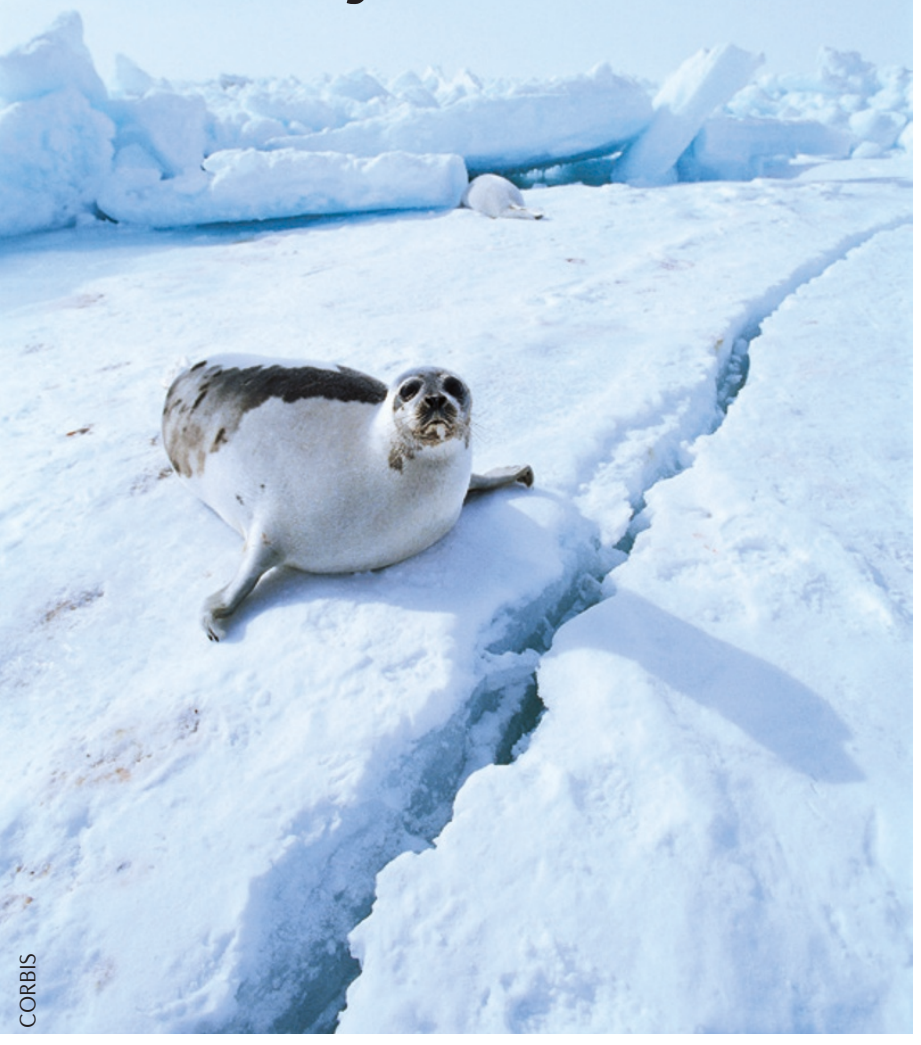

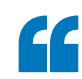

a highly sensitive

and selective

transcriptional

response to

the first few

interacting

symbionts
The early interactions between a bacterial symbiont and its host are crucial for establishing a long-lived association, but the molecular mechanisms underlying this initial dialogue are poorly understood. Using the well-established Vibrio fischeri-squid symbiosis as a model system, Kremer et al. now show that the few $V$. fischeri cells which initiate the association induce transcriptional changes in the host, and these changes prime the symbiont for subsequent colonization.

The exclusive partnership between $V$. fischeri and the Hawaiian bobtail squid (Euprymna scolopes) is lifelong, but it must be reconstituted in the light organ of each juvenile squid after hatching in the ocean. This complex process involves initial attachment of just 3-5 V. fischeri cells to the mucociliary epithelium on the exterior of the light organ, followed by aggregation of this population at the light organ pores and subsequent migration into the crypts of the light organ, where they proliferate. Enrichment of $V$. fischeri from the highly diverse marine bacterial community has previously been shown to depend on epithelial mucus, including the production of a gradient of chitobiose (a chitin breakdown product), which promotes colonization.

To determine whether changes in host gene expression accompany initiation of the symbiosis, Kremer et al. compared the transcriptome of symbiotic light organs (which were exposed to thousands of environmental bacteria and an average of five $V$. fischeri cells) to that of aposymbiotic light organs (which were exposed to environmental bacteria only). The results revealed that protease and hydrolase transcripts were increased in the presence of the few associating $V$. fischeri cells, particularly those associated with chitin metabolism and most notably the gene encoding chitotriosidase.

Chitotriosidase from other species is a secreted enzyme that cleaves chitin polymers and releases chitobiose, among other by-products. Purified E. scolopes chitotriosidase also showed catalytic activity, which was optimal at the acidic $\mathrm{pH}$ of the epithelial mucus.
Furthermore, immunomicroscopy localized the encoding transcript and the protein to regions of the light organ where $V$. fischeri accumulates, and chitin by-products were enriched at the pores of the light organ, where $V$. fischeri senses the chitobiose gradient.

After aggregation at the pores, but before migration into the crypt, $V$. fischeri cells seem to pause as they prepare or are 'primed' for new environmental conditions, such as a chemoattractant gradient. Kremer et al. showed that pre-exposure of $V$. fischeri to chitobiose enhanced migration along a chitobiose gradient. This suggests that early exposure of aggregated cells to chitobiose (generated by the action of chitotriosidase) at the light organ pore primes them for migration into the crypts.

Collectively, these findings define the molecular mechanisms and spatiotemporal dynamics underpinning the initial events that lead to the establishment of the $V$. fischeri-squid symbiosis. The mucociliary epithelium of the light organ has evolved a highly sensitive and selective transcriptional response to the first few interacting symbionts, which results in a change in the local chemical environment that promotes subsequent colonization. This study illuminates the key molecular strategies required to initiate a beneficial symbiosis, the principles of which are likely to be widespread.

Christina Tobin Kåhrström

ORIGINAL RESEARCH PAPER Kremer, N. et al. Initial symbiont contact orchestrates host-organwide transcriptional changes that prime tissue colonization. Cell Host Microbe 14, 183-194 (2013) FURTHER READING Nyholm, S. V. \& Graf, J. Knowing your friends: invertebrate innate immunity fosters beneficial bacterial symbioses. Nature Rev. Microbiol. 10, 815-827 (2012) 